\title{
Demand Response by Decentralized Device Control Based on Voltage Level ${ }^{\star}$
}

\author{
Wilfried Elmenreich ${ }^{1,2}$ and Stefan Schuster ${ }^{1}$ \\ 1 Dep. of Informatics and Mathematics \\ University of Passau, Germany \\ 2 Netw. and Emb. Systems/Lakeside Labs \\ Alpen-Adria-Universität Klagenfurt, Austria \\ wilfried.elmenreich@uni-klu.ac.at
}

\begin{abstract}
This paper introduces a distributed, self-organizing approach to load control based on voltage measurement. A local voltage measurement defines a Level of Service (LoS), which is balanced with the neighboring households in order to avoid extreme restrictions of energy use in a single household. The approach distinguishes four criticality classes for devices which suspend themselves at specific LoS thresholds.
\end{abstract}

\section{Introduction}

The amount of alternative energy sources such as photovoltaic systems or wind power will increase significantly in future. Renewable energy sources, however, typically rely on the weather and thus lead to variable energy production which is hard to manage [1]. In this work, we concentrate on how to handle power under-supply, i.e., the situation where power demand exceeds possible production capability. Based on the ColorPower architecture described in [2], we propose a decentralized control mechanism to temporarily turn off devices dependent on their criticality. Although in 2 all modeled devices consume an equal amount of energy, we consider devices with a wider range of power consumption, targeting typical domestic application. Following [3], we organize devices into households and take effects such as connection resistivity into account.

\section{Device Model}

A user or given schedule decides when a device is needed. This is modeled by a starting time $t_{o n}$ and a duration of use, $d_{o n}$, which are both normally distributed with standard deviations $\sigma_{t_{o n}}$ and $\sigma_{d_{o n}}$, respectively. Requests for using a device are typically periodically with period $T$. As an example, the parameters for a stove could look like this: $t_{o n}=11 A M, \sigma_{t_{o n}}=0.5, d_{o n}=0.75, \sigma_{d_{o n}}=0.3$,

\footnotetext{
* This work is supported by the Carinthian Economic Promotion Fund (KWF) under grant 20214/22935/34445 (Project Smart Microgrid Lab). We would like to thank Lizzie Dawes for proofreading the paper.
} 
$T=24 h$, i.e., the stove is used daily around $11 A M$ for about $45 \mathrm{~min}$. A device currently requested is called requested. As long as the energy provided is sufficient, all requested devices are turned on, i.e., they are active. If a device is requested but cannot be turned on because of energy shortage, it is suspended. Depending on their criticality, devices are classified into four categories identified by the colors green, blue, red and black based on the ideas in 2. "Green" devices can be suspended without disturbing the user significantly and without losing vital services. An example of a green device is a washing machine programmed by the user to wash within the next few hours, but not necessarily immediately. "Blue" devices are not vital to the infrastructure, but annoy the user if they are not working, e.g., supplemental lighting systems. "Red" devices significantly affect the user, for example food in a refrigerator will spoil faster if the refrigerator is turned off. "Black" devices are the most vital services for the infrastructure that should be maintained operational as long as possible.

\section{Device Control by Voltage Level}

The voltage level can be used as a measure for the amount of energy available in the system; voltage drops if not enough energy can be supplied. The standard IEC 60038 defines valid voltage as $\pm 10 \%$ of the nominal voltage.

Devices in the household can counteract a supply shortage by reducing demand upon a voltage drop. This is done by suspending devices.

Figure 1 describes a mapping of voltage levels to the four types of criticality classes or labels. A particular voltage level defines which device classes have to be turned off completely, which classes are unaffected, and which device class is partially suspended.

\begin{tabular}{|c|c|c|c|c|}
\hline $\mathrm{V}$ & $230 \mathrm{~V}$ & $225 \mathrm{~V}$ & $220 \mathrm{~V}$ & $215 \mathrm{~V}$ \\
\hline LoS & {$[4.0-3.0)$} & {$[3.0-2.0)$} & {$[2.0-1.0)$} & {$[1.0-0.0]$} \\
\hline green & $\mathrm{s}$ & $\mathrm{o}$ & $\mathrm{o}$ & $\mathrm{o}$ \\
blue & - & $\mathrm{s}$ & $\mathrm{O}$ & $\mathrm{o}$ \\
red & - & - & $\mathrm{s}$ & $\mathrm{o}$ \\
black & - & - & - & $\mathrm{s}$ \\
\hline
\end{tabular}

Fig. 1. Exemplary mapping between labels and voltage levels. For example, if the voltage is between 220 and $225 \mathrm{~V}$, part of the blue devices are suspended (s), below $220 \mathrm{~V}$ all blue devices are turned off (o).

Figure 2 shows the possible states and state transitions of a device. If a user wants to turn on a device, the device goes into state on-protected (unless the voltage level does not allow at that time for using that class of devices - if this is the case, the device goes into the state suspended-protected). A state with addition protected means that the device will stay in this state for a predefined amount of time in order to avoid devices switching on and off repeatedly. Partial suspension of a device class is implemented by a probabilistic algorithm. Based on the function in Figure2, a particular probability level $p$ defines the probability 


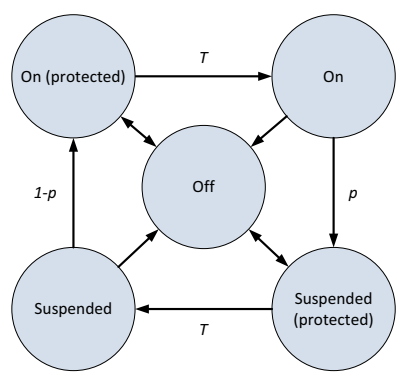

Fig. 2. Device control states

of a device being suspended. After the protection time $T$, a device can come back on with the reverse probability $1-p$. After some time, this algorithm converges towards having a ratio $p$ of suspended devices.

\section{Fairness by Synchronization}

The voltage drop gives good feedback on the local grid situation, however, due to different cable lengths, there are likely to be voltage differences between neighboring houses. To ensure fairness, we require that within a small distance, e.g., from one house to another, the amount of suspended devices should not differ significantly. On the other hand, if a larger area, e.g., a whole neighborhood, experiences a voltage drop, the consumers in the area should collectively react to the problem. Therefore, we introduce a layer between measured voltage and mapping in Figure 1 named "Level of Service" (LoS). The LoS is repeatedly calculated from the local measured voltage level and an averaging process based on the neighbors' communicated LoS as follows:

1. LocalLoS $=f($ Voltage $)$

2. Receive LoS values from neighbors via local wireless communication

3. $L o S=\alpha \cdot$ average $\left(\right.$ neighbor $\left.s^{\prime} L o S\right)+(1-\alpha)$ LocalLoS

4. Broadcast LoS

The coupling factor, $\alpha$, determines how much the LoS is influenced by the situation in the neighborhood.

\section{Simulation}

For evaluation we simulate up to 10 streets, each consisting of 20 to 50 households linked together and connected to a power provider (e.g., a mid/low voltage transformer). The wireless network for synchronization is based on a CC2420 radio with a transmission range of approximately $30 \mathrm{~m}$. The model is based on [3]. The model accounts for the resistance of cables between households. These resistances are derived from physical and electrical properties of typical feed cables 
(street cable $150 \mathrm{~mm}^{2}$, building connection $35 \mathrm{~mm}^{2}$ ). We implement a simple energy flow model without reactive loads. The energy consumption of each household is modeled via a number of devices, which are modeled according to the device model described in Section 2 We randomly assigned up to $\pm 10 \%$ of the required energy for each street, causing energy shortages in some of the streets. If the total load of households in a street corresponds to higher power than provided, this results in a lower voltage at the feed and, consequently, in reduction of power consumption proportional to $\left(\frac{U_{\text {nominal }}-U_{d r o p}}{U_{\text {nominal }}}\right)^{2}$ for ohmic devices. The left table in Figure 3 shows the LoS map based on the local voltage in each house. The feed is from left to right, so LoS are generally higher on the left side. There are significant differences depending on the respective feed section. The right part of Figure 3 shows the LoS after update with the synchronization algorithm with a coupling factor $\alpha=0.5$.
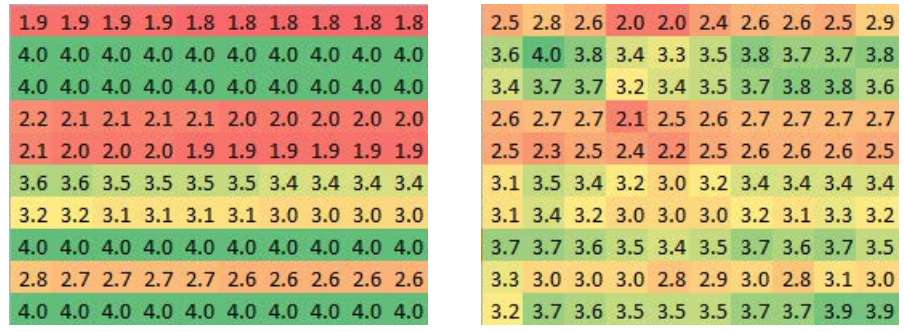

Fig. 3. Level of Service before and after synchronization

\section{Conclusion}

We presented a distributed, self-organizing approach to load control based on voltage measurement. A local wireless network and a synchronization algorithm are used to improve fairness between neighboring consumers. The approach is suited to handle networks with supply shortages, thus is especially of interest for islanded grids with power sources from alternative energy such as photovoltaic systems or wind power. For a fine-grained demand response, each house needs smart appliances which can be controlled by the presented system. Future work will include simulations with improved appliance models and heterogeneous distributions of houses.

\section{References}

[1] Sobe, A., Elmenreich, W.: Smart microgrids: Overview and outlook. In: Proc. of the ITG INFORMATIK Workshop on Smart Grids, Braunschweig, Germany (2012)

[2] Beal, J., Berliner, J., Hunter, K.: Fast precise distributed control for energy demand management. In: Proc. of the Sixth IEEE International Conference on Self-Adaptive and Self-Organizing Systems, Lyon, France, pp. 187-192 (2012)

[3] Okeke, I.O.: The influence of network topology on the operational performance of the low voltage grid. Master's thesis, Delft University of Technology (2012) 